\title{
Observation of Ballooning Instabilities with Medium Toroidal Mode Number in
} High Temperature Tokamak Plasmas

PPFL- -2850

DE92 018962

Y. Nagayama ${ }^{a)}$, S. A. Sabbagh ${ }^{\text {b.c })}$, J. Manickam, E. D. Fredrickson, M. Bell, R.V. Budny, A. Cavallod), A. C. Janos, M. E. Mauel ${ }^{\text {b), }}$ K. M. McGuire, G. A. Navratil ${ }^{b)}$, G. Taylor, and M. Yamada

Princeton Plasma Physics Laboratory, Princeton University Princeton, New Jersey 08543

\section{Abstract}

A beta degradation event in a high $\beta_{p}$, TFTR tokamak plasma is analyzed with X-ray and electron cyclotron emission (ECE) imaging techniques. Medium-n (toroidal mode number) instabilities with ballooning characteristics are observed near and within the $q=1.5$ surface during a slow degradation in the plasma $\beta$ and precede a sudden-partial collapse in the central plasma pressure. This is the first reported observation of a ballooning instability in the interior of a large, collisionless tokamak plasma.

\footnotetext{
a) Present address: University of Tsukuba, Ibaraki 305, Japan

b) Columbia University, New York, N.Y. 10027

c) Present address: Plasma Physics Laboratory, Princeton University

d) Present address: Center for Energy and Environment Studies, Princeton University
} 
The economic feasibility of tokamaks as fusion reactors is strongly dependent on the maximum value of $\beta$ (the ratio of plasma to magnetic field pressure) that can be achieved in these devices. The $\beta$ in present tokamak experiments is limited by fast disruptions that terminate the discharge, or by instabilities that either suddenly or gradually degrade the plasma confinement. Empirically, it has been found that the maximum $\beta$ obtained in tokamaks is consistent with the Troyon limit [1], $\beta_{\max }=3 \times 10^{-8} I(\mathrm{~A}) / a(\mathrm{~m}) B(\mathrm{~T})$, which is derived from a combination of experimental data and theoretically predicted instability thresholds for ideal magnetohydrodynamic (MHD) kink and ballooning modes. However, ballooning instabilities, which theoretically have fast growth times $(\sim 10 \mu \mathrm{s})$ and short wavelengths perpendicular to the magnetic field, have not yet been observed and identified in large tokamak experiments.

A significant result of the present work is the observation of medium-n, $(4 \leq n \leq 10)$ ballooning modes that appear in a series of discharges in TFTR designed to achieve high $\beta_{p}\left(\beta_{p} \geq 2\right)$ operation [2]. The modes occur during a slow degradation in the plasma $\beta$ and precede a sudden partial collapse in the central plasma pressure. The amplitude of the mode is larger on the outboard (low-field) side of the torus and has a fast growth rate, characteristic of an ideal MHD ballooning instability. While high-n ballooning modes were observed in a small, short pulselength resistive plasma in TORUS II [3], this is the first reported observation of a ballooning mode in the interior of a large, collisionless tokamak plasma.

Figure 1 shows the time evolution of plasma parameters for two high $\beta_{p}$ plasmas, one with, and one without a degradation in poloidal beta. The plasmas were created by rapidly decreasing the plasma current prior to neurral beam injection. The discharge heated with $18 \mathrm{MW}$ of power (shot 54018) experiences a gradual degradation in $\beta_{p}$ beginning at $t=3.77 \mathrm{~s}$, and a sudden collapse in the central density at $t=3.78 \mathrm{~s}$. MHD oscillations are also greatly enhanced at this time, leading to a reduction in plasma confinement. The other discharge shown does not 
suffer a similar reduction in plasma parameters. This plasma has the same evolution of total current, but is heated with only $15 \mathrm{MW}$ of neutral beam power.

The time evolution of the electron temperature profile and the soft $X$-ray emission profile at the moment of the $\beta$ collapse is shown in Fig. 2. The electron cyclotron emission (ECE) is measured with a 20 channel grating polychromator which is cross-calibrated to a Michelson interferometer [4]. The data is collected with a $2 \mu \mathrm{s}$ time resolution covering the major radius from $R=2.3-3.3 \mathrm{~m}$. The channel separation is $6 \mathrm{~cm}$ with a radial resolution of $3 \mathrm{~cm}$. The soft $X$-rays are detected with a vertical camera (20 detectors) and a horizontal camera (60 detectors) [5]. It is useful to subtract the time averaged part from the total ECE signal and view the perturbation profile, Fig. 2(b). The contour plot of the perturbation amplitude provides a visual representation of the instabilities and facilitates identification of the location and relative spatial intensity of the oscillations [6].

The slow degradation in $\beta_{p}$ begins at $3.7 \mathrm{~s}$ with the growth of an $(m, n)=(3,2)$ mode. The $(3,2)$ mode has only a weak effect on confinement. About $80 \mathrm{~ms}$ later, (at $t=3.7799 \mathrm{~s}$ ) a fast drop in the central electron temperature occurs. Hereafter, we will reference times from $t=3.7795 \mathrm{~s}$, defining $\Delta t=t-3.7795 \mathrm{~s}$. A second, higher frequency mode starts to grow at $\Delta t=0.14 \mathrm{~ms}$. These modes are located near the radius of the $(3,2)$ mode. After the onset of the higher frequency mode, the MHD activity expands inward. At $\Delta t=0.3 \mathrm{~ms}$, a strong $n=6$ mode appears and the flattening of the temperature profile near the $q=1.5$ surface is completed. During the crash $(\Delta t=0.35-0.43 \mathrm{~ms})$, a $(1,1)$ mode appears and the central electron temperature decreases. Following the crash, the $(3,2)$ mode appears along with the $(1,1)$ mode. The medium $n$ mode has a growth time of less than $20 \mu \mathrm{s}$. This last mode shows a strong inside/outside asymmetry with a larger amplitude on the weak field side as might be expected for a ballooning mode [see Figs. 2 and 3(e)]. A similar instability has been observed in other TFTR discharges 
(about 20) for which the fast time resolution ECE data has been analyzed. A summary of a few of these shots is given in Table 1.

The ballooning mode is not detectable on the Mirnov coil system, thus the usual method of identifying mode numbers cannot be used. Identification of the toroidal mode number is made by taking advantage of the 180 separation of the horizontal X-ray camera and the grating polychromator. Comparison of the ECE profile and the horizontal X-ray profile gives $n=6 \pm 1$.

The poloidal mode number of a helical instability in a tokamak plasma can be estimated by simulating the X-ray emission of a perturbed model source function [7]. By rotating the model $\mathrm{X}$-ray source function toroidally at the proper frequency, the simulated signals can be compared with the actual signals to obtain the $m$ number for the observed $n=6$ instability (Fig. 3). The experimental data describing the initial growth of the mode is well modelled by invoking a helical "wave packet" perturbation that is localized in the poloidal and toroidal directions. The best agreement is obtained between the simulation and experiment when a perturbation with $m=7 \pm 2$ is used [see Fig. 3(b, d)], but whose amplitude is significant over approximately $3 / 7$ of the poloidal cross section. The terminology " $(m, n)=(7,6)$ wave packet" means that the instability has a $(7,6)$ helical structure. This source function model is shown in various phases in Fig. $3(\mathfrak{f}, g)$ corresponding to the times shown in Fig. 3(b, d). Obtaining agreement with the measured signals also requires that the Shafranov shift of the magnetic axis is included and that the localized perturbation amplitude of the mode increases when it resides on the weak field side of the torus [shown in Fig. 3(g)], characteristic of a ballooning instability. The maximum peak-to-peak amplitude of the experimentally measured soft X-ray fluctuation level [Fig. 3(e)] exhibits a similar ballooning feature.

These discharges have been modelled using the TRANSP code [8]. The equilibrium representing the plasma prior to the degradation in $\beta_{p}$ was computed by a two-dimensional flux-coordinate equilibrium solver 
using the radial pressure, $q$ profiles and outer boundary information taken from the TRANSP simulation. At $t=3.75 \mathrm{~s}$, the central $q$ value was 1.28 , the edge $q$ was 14.7 , and a region of low shear was present near $q=1.5$. The pressure profile was peaked, with the ratio of central to volume averaged pressure being 5.5. Although the simulated profiles near the magnetic axis are uncertain, the absence of sawtooth activity gives credence to modelling $q_{0}>1$.

The stability to both kink and ballooning modes was computed for these equilibria. It is found that prior to the sharp drop in the central pressure, the plasma is close to the threshold for instability to high-n ballooning modes. The computed equilibrium remains stable (near marginal stability) when $q_{0}$ is reduced from 1.28 to 1 [Fig. 4(a)]. Stability to low- $n$ ideal MHD modes was determined using the PEST code [9]. The plasma was computed to be stable to the $n=1$ external kink mode and unstable to an $n=6$ internal ballooning mode. The peaked pressure and low shear near the magnetic axis suggest that the instability is of the infernal type [10]. This was verified by examining the stability while varying the toroidal mode number. The dependence of the growth rate on $n$ has the oscillatory behavior typical of infernal modes. A similar resonant behavior is expected if $q_{0}$ is varied, with different $\mathrm{m} / \mathrm{n}$ numbers being unstable [Fig. 4(b)]. The instabilities have predicted growth-times in the range of $10 \rightarrow 50 \mu \mathrm{s}$, which compare quite well to the observed values.

A major difficulty in equilibrium and stability analysis of this type is the experimental uncertainty in the plasma pressure profile (especially the nonthermal component) and the $q$ profile. Efforts to obtain detailed measurements of the equilibrium $q$ profiles will be carried out in the future to improve the comparison between theory and experiment.

In summary, ballooning modes of medium- $n$ value have been observed in high $\beta_{p}$ plasmas with pressure gradients calculated to be near the first stability boundary. The following observations support the identification of these modes as ballooning modes: (1) the identified toroidal mode numbers $(4 \leq n \leq 10)$ are much higher than the usual kink or 
tearing mode, (2) the modes are stronger on the weak field side of the torus (i.e., they exhibit a strong ballooning characteristic), (3) the growth rate of the modes is consistent with the calculated ballooning mode growth rate using linear ideal MHD stability theory, and (4) reconstruction of the plasma equilibrium shows the pressure gradient to be near the first stability boundary and unstable to infernal-type ballooning modes.

\section{Acknowledgements}

The authors would like to thank Dr. M. Phillips of the Grumman Corporation for the use of equilibrium and stability codes. Dr. H. Park for the electron density measurement, and Drs. M. Chance, M. Okabayashi and W. Park for useful discussions. This work was supported by the U.S. Department of Energy under contract No. DE-AC02-76-CHO-3073 and DEFG02-89ER53297. 
References

[1] F. Troyon et al., Plasma Physics and Controlled Fusion 26, 209 (1984).

[2] S. A. Sabbagh et al., Phys. Fluids B 3, 2277 (1991).

[3] M. Machida and G.A. Navratil, Phys. Rev. Lett. 51, 992 (1983).

[4] A. Cavallo et al., Rev. Sci. Instrum. 59, 889 (1988). F. J. Stauffer et. al., ibid. 59, 2139 (1988).

[5] K. W. Hill et al., Rev. Sci. Instrum. 56, 830 (1985). L. C. Johnson et al., ibid. 57, 2133 (1986).

[6] Y. Nagayama et al., Phys. Rev. Lett. 67, 3527 (1991).

[7] S. Tsuji et al., Nucl. Fusion 25, 305 (1985).

[8] R. J. Hawryluk, in Physics of Plasma Close to Thermonuclear Conditions, edited by B. Coppi et al., (Commission of the European Communities, Brussels, 1980), Vol.1, p.19.

[9] R. Grimm, R.L. Dewar and J. Manickam, J. Comput. Phys. 49, 94 (1983).

[10] J. Manickam, N. Pomphrey and A. M. M. Todd, Nucl. Fusion 27, 461 (1987). 


\section{Figure Captions}

FIG. 1.

Summary of the time dependence of various plasma parameters for two high $\beta_{p}$ discharges. The solid line indicates the beta degradation case (shot 54018 ) and the broken line indicates a case in which $\beta_{p}$ saturates (shot 54013). The vertical arrow indicates the time at which the ballooning mode is observed.

FIG. 2.

Contour plots of the time evolution of the ECE profile in shot 54018: (a) electron temperature profile; the contour step size is $300 \mathrm{eV}$, (b) perturbation of ECE signal, (c) chord integrated soft X-ray emission profile (horizontal view).

FIG. 3.

Comparison of observed soft X-ray signals and the signals simulated by the model source function: (a) the observed soft $X$-ray signals from the horizontal camera, (b) the simulated waveforms from the horizontal camera by the $(m, n)=(7,6)$ model, (c) the observed soft $X$-ray signals from the vertical camera, (d) the simulated waveforms from the vertical camera by the $(7,6)$ model, (e) maximum peak-to-peak amplitude of the fluctuation between $3.77977 \mathrm{~s}$ and $3.77984 \mathrm{~s}$, where the hatched area is the base fluctuation level which is sampled between $3.7794 \mathrm{~s}$ and $3.7796 \mathrm{~s}$, (f) "wave packet" model source function at time "A", ( $\mathrm{g}$ ) "wave packet" model source function at time "B". The times "A" and "B" are shown in frames (b) and (d). Note that the amplitude of the perturbation has increased as it has moved to the weak field side of the torus.

FIG. 4.

(a) High- $n$ ballooning stability for high- $\beta_{p}$ discharge 54018 . The solid line indicates the equilibrium pressure gradient at $t=3.75 \mathrm{~s}$. The enclosed 
region is unstable to high- $n$ ballooning modes. The pressure gradients are plotted vs. the square root of the normalized poloidal flux, which corresponds to the minor radius. (b) Growth rate of infernal modes with various toroidal mode numbers vs. $q_{0}$. The values are normalized to the poloidal Alfven frequency. 


\begin{tabular}{|llllll|}
\hline TFTR & \multicolumn{3}{c|}{ precursor } & \multicolumn{2}{c|}{ Ballooning mode } \\
Shot No. & $\beta_{p}$ & $q_{a}$ & $(m, n)$ & $n$ & $\tau(\mu \mathrm{s})$ \\
\hline \hline 44677 & 1.3 & 5.9 & not clear & 4 & 50 \\
53356 & 1.6 & 4.9 & $(3,2)$ & 6 & 80 \\
54017 & 1.9 & 7.5 & $(4,3)$ & 3 & 20 \\
54018 & 2.1 & 7.6 & $(3,2)$ & 6 & 30 \\
54021 & 2.2 & 7.5 & $(3,2)$ & 6 & 20 \\
\hline
\end{tabular}

TABLE I. Summary of discharges in which medium- $n$ modes are observed. The toroidal mode number of the ballooning instability, the growth time and the precursor mode are given, along with the plasma edge safety factor and $\beta_{p}$. The mode growth time generally decreases as the $\beta_{p}$ increases. 

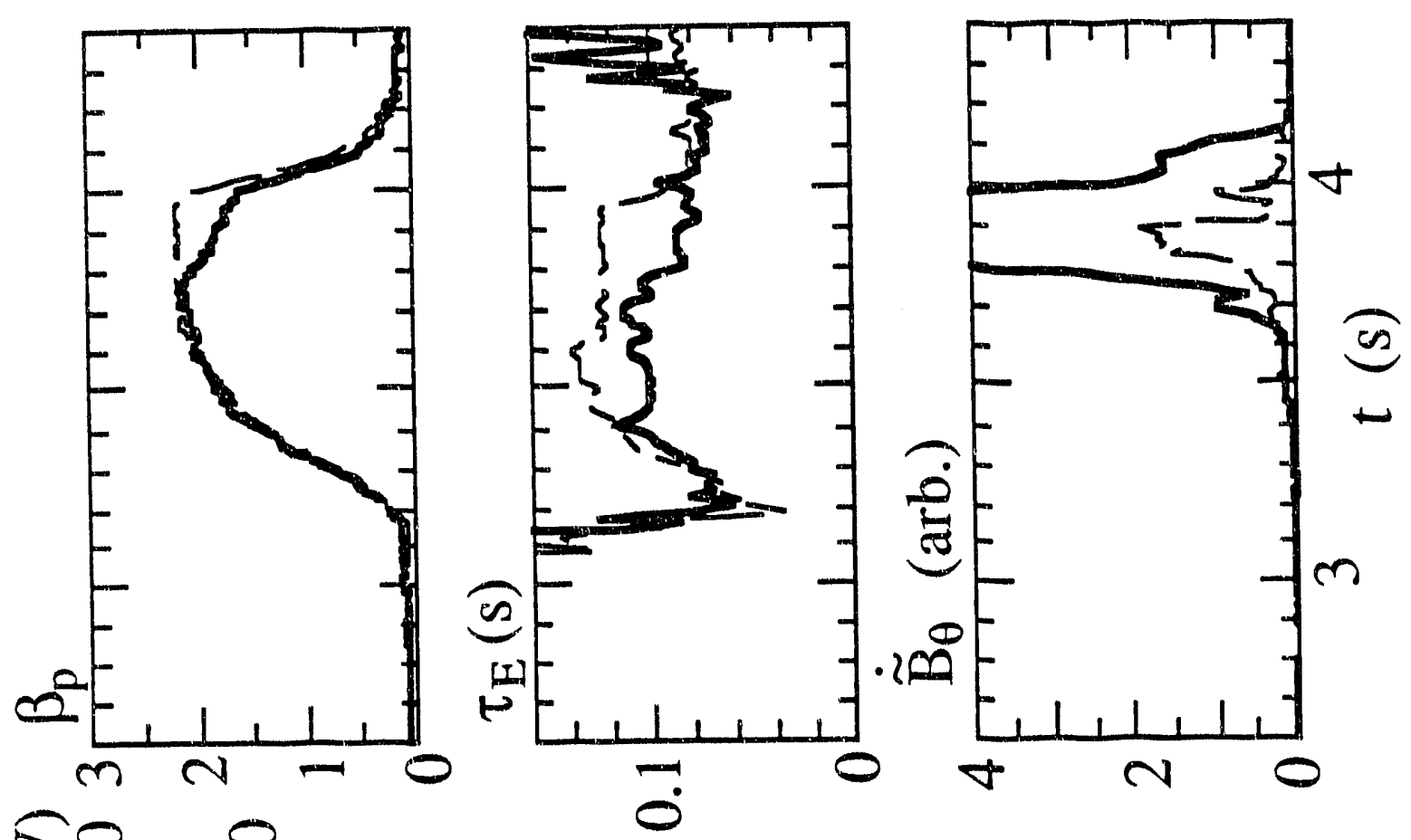

$-1$
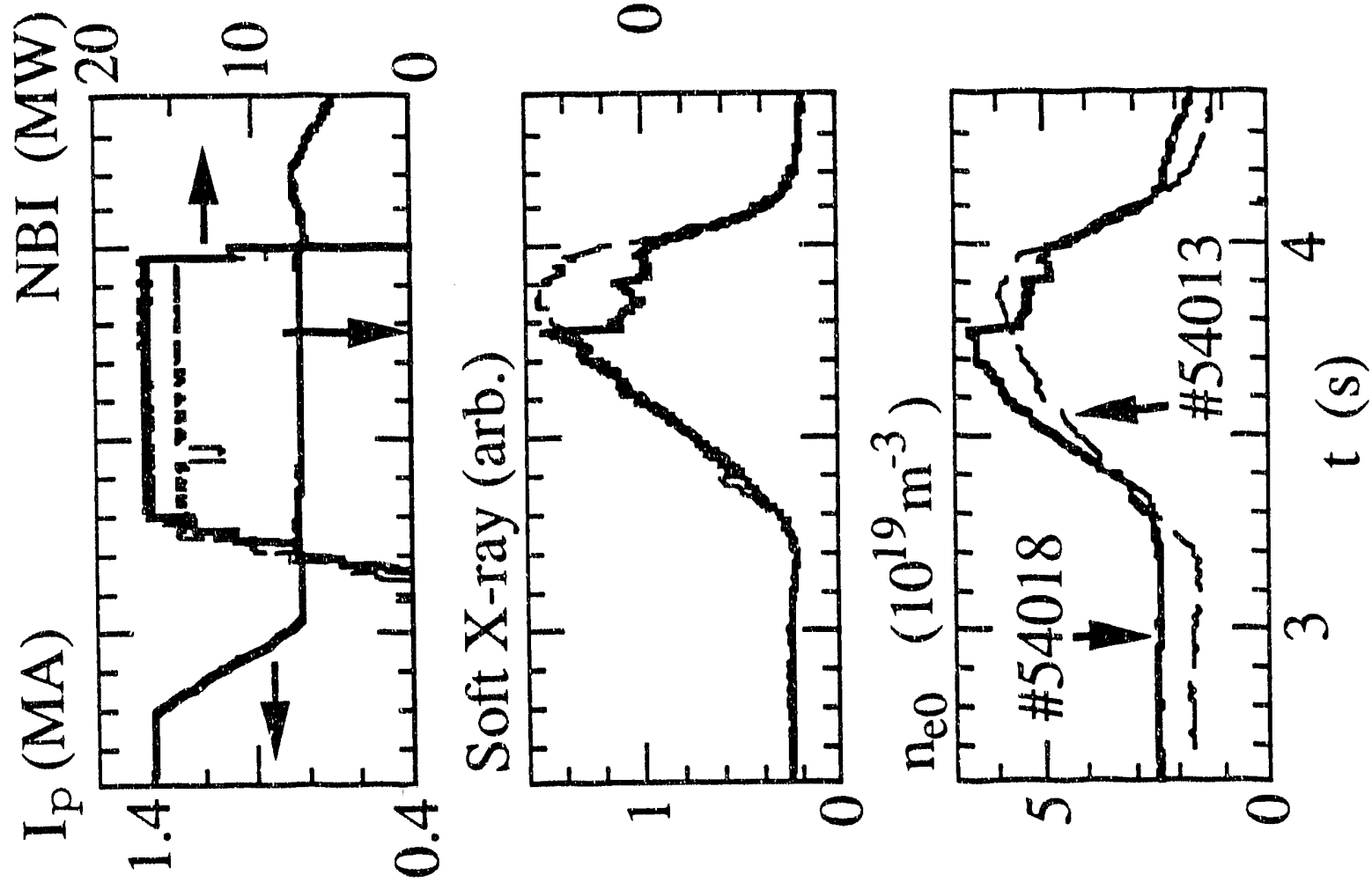
(a) ECE (total)

shot 54018

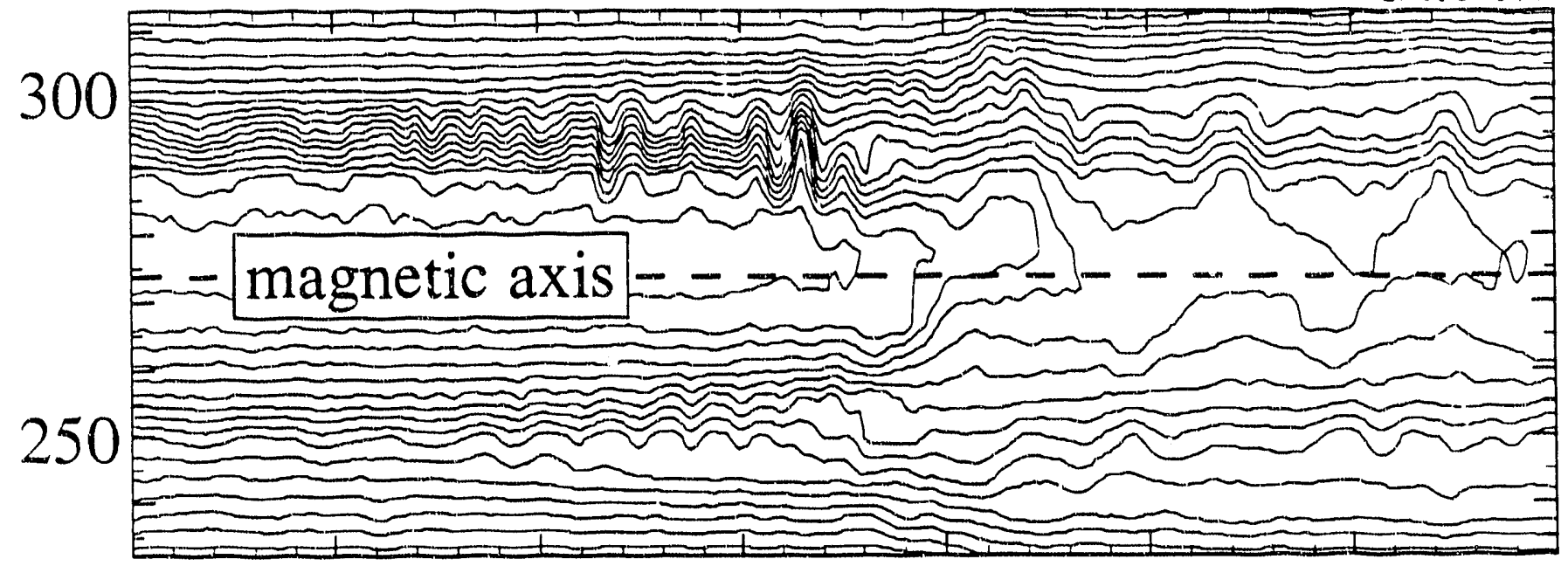

(b) $\mathrm{ECE}$ (perturbation) $(7,6)$ $300=1.50$ (1)

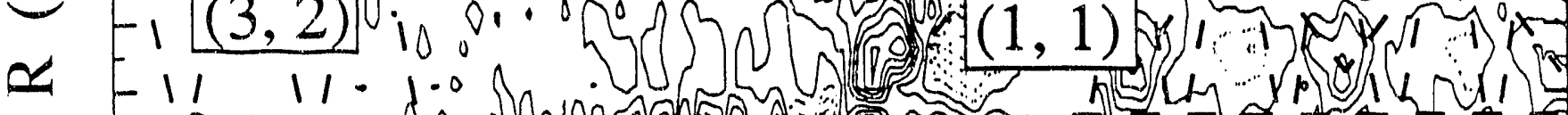

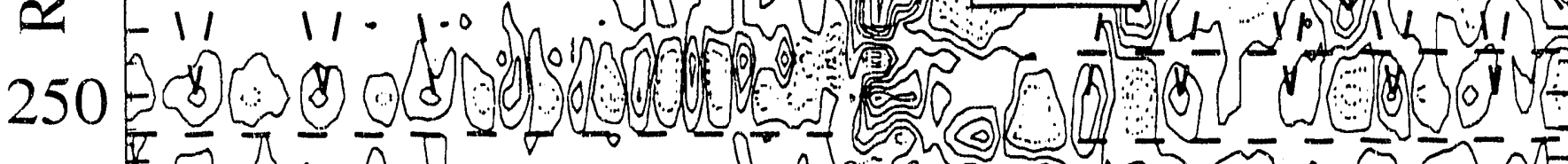

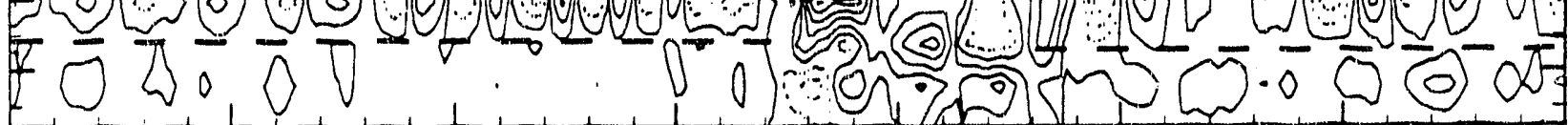

(c) soft X-ray (horizontal view)

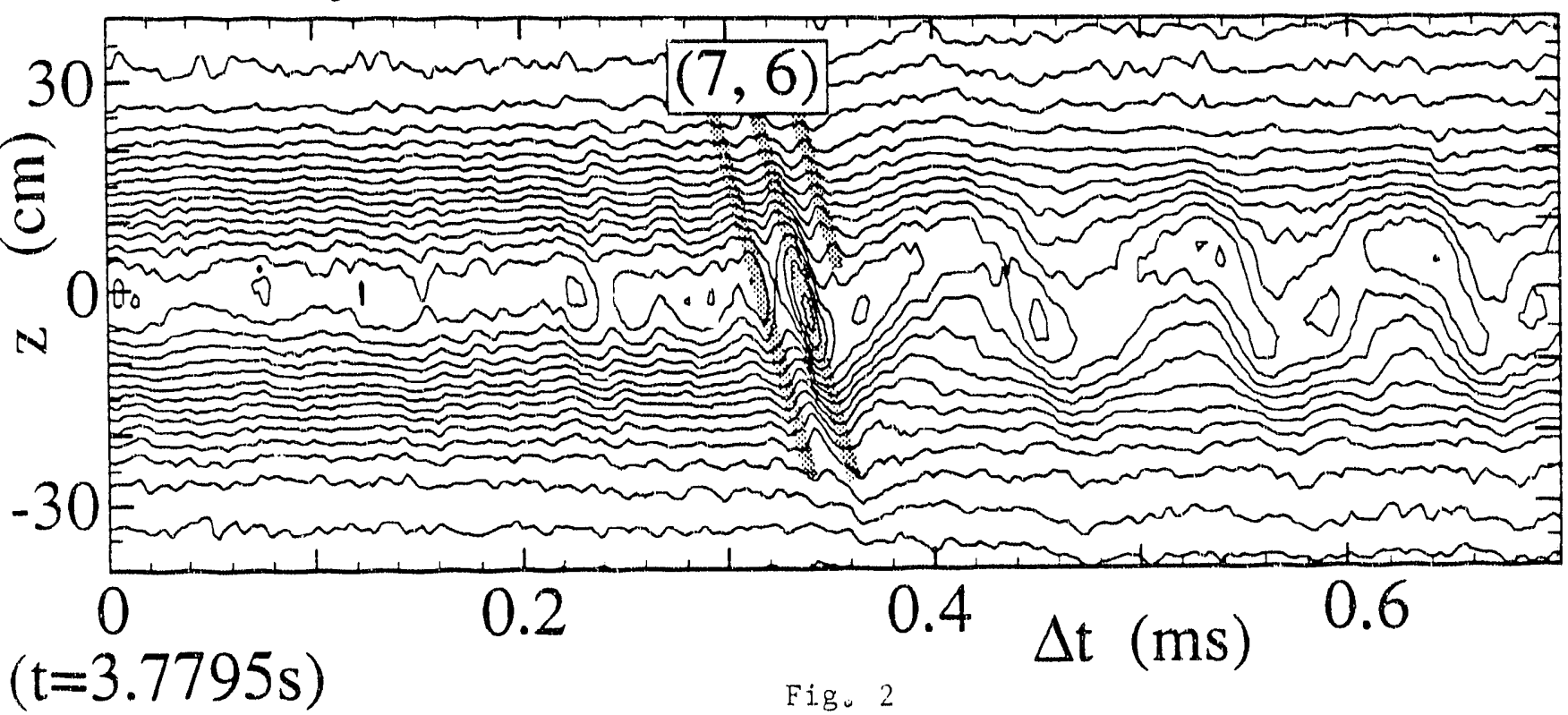



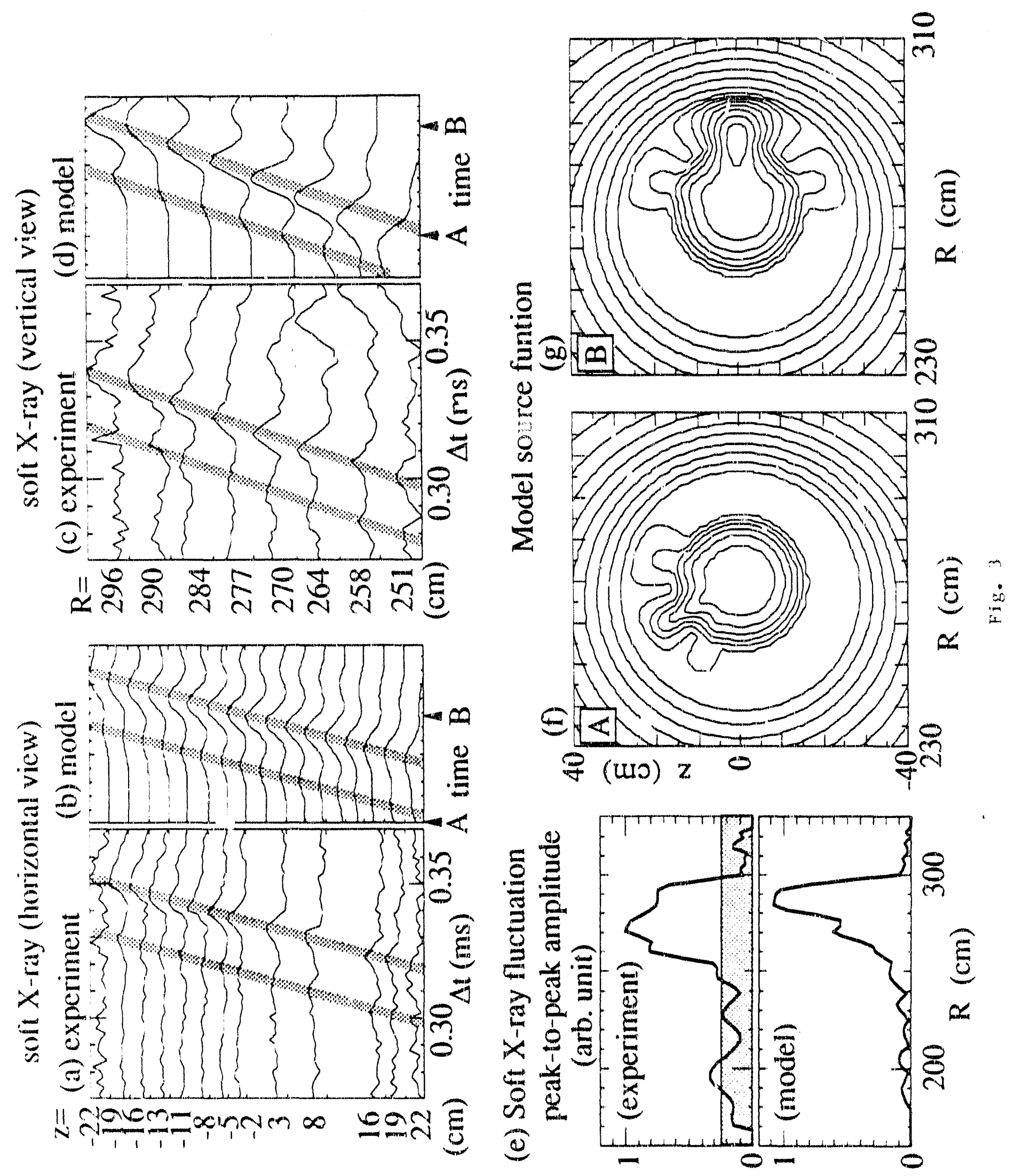

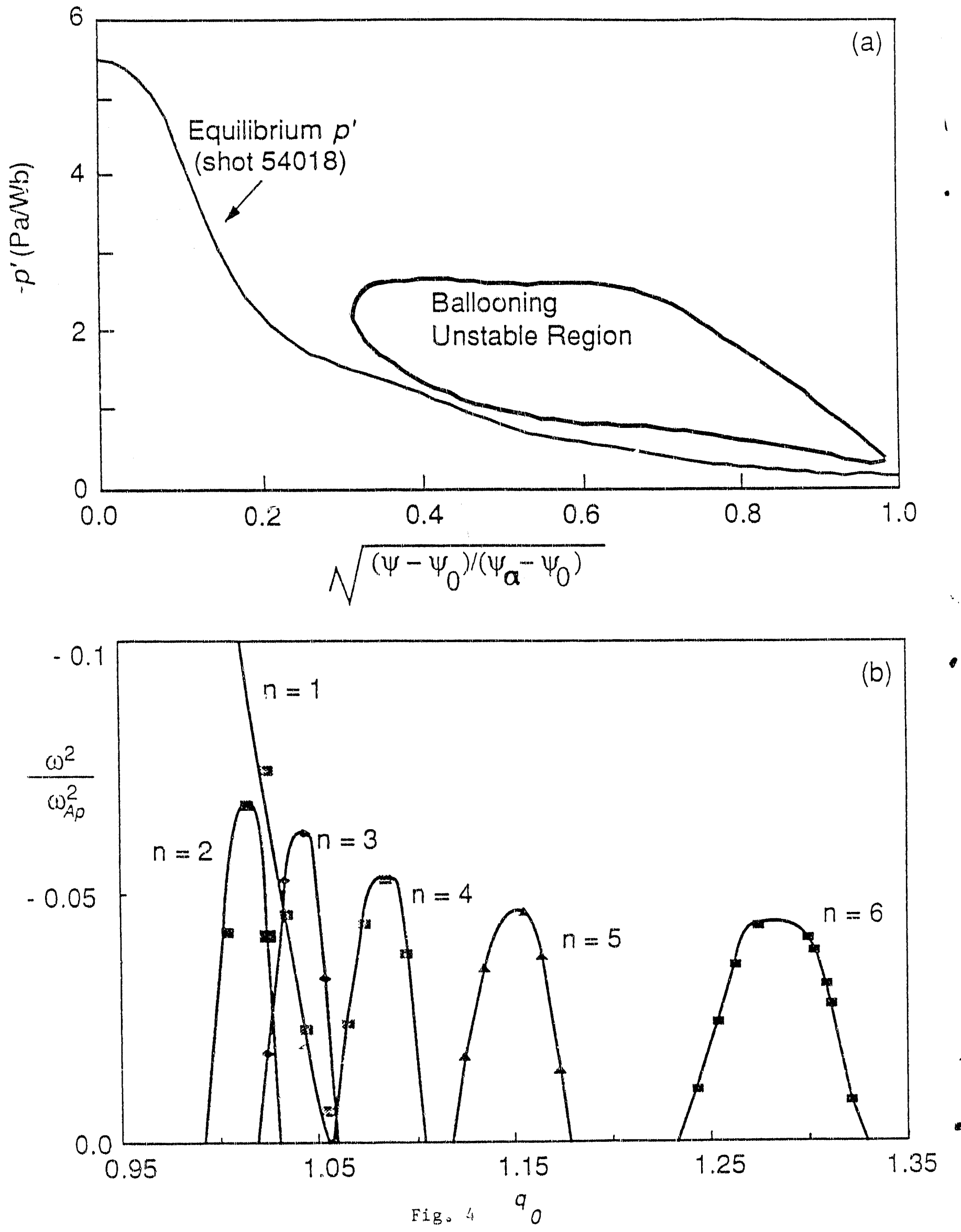
Dr. F. Paoloni, Univ. of Wollongong, AUSTRALIA

Prot. M.H. Brenran, Univ. of Syorroy, AUSTRALIA

Plasema Rosearch Leb., Austration Nat Univ., AUSTRAL.IA

Prol. I.A. Jones, Findors Univ, AUSTRALIA

Prof. F. Cap, Inst for Theoretical Physics, AUSTRIA

Prot. M. Hoinderer, Instwit hor Theoretieche Physik, AUSTRIA

Prot. M. Gooseen, Actronomiect Instibsut, BELGIUM

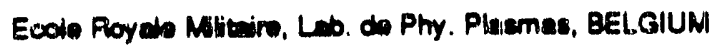

Comminsion-Europaen, DG. XII-Fueion Prog., BELGIUMA

Prof. F. Bowciqub, Ritheuniverwitis Gent, BELGIUM

Dr. P.H. Sakanaka, Iraitituto Ficica, BRAZIL

In tuturto Necioned Do Puspican Eapediair-INPE, BRAZIL.

Documents Orico. Alomic Enworgy of Cenedil Lid., CAMADA

D. M.P. Bestrynakj, MPB Technologios, Inc., CANADA

Dr. H.M. Stkaragara, Univ. of Sastuatchewen, CAALADA

Prof. J. Toichmann, Univ. of Montred, CANADA

Prot. S.R. Sromivonen, Univ. of Celoger, CANADA

Prof. T.W. Jolmetton. INRS-Energio, CANADA

Dr R. Botton, Centre canadian de husion mapndique, CANADA

Dr. C.R. Jemos., Univ. of Aborte, CANMOA

Dr. P. Lukde, Komchutho Universzit, CZECHOSLOVAKIA

The Librarien, Cutram Laborabry, ENGLANO

Librery, A61, Puthartord Appletion Laboratory, ENGLAND

Mrt. S.A. Murchineon, JET Librey, ENGLAND

Dr. S.C. Sherma Univ. of South Pecific, Flul ISLANOS

P. Muthonen, Univ. Haleinki, FINLAND

Prof. M.N. Buacec, Ecol Potyticturique., FRANCE

C. Moutter, Lob. do Phyaique doe Milioux loniads, FRANCE

J. Rodet CENVADARMCHE - Bat 506, FRANCE

Prot. E. Economou, Univ. of CreAs, GREECE

As. C. Pinni, Univ. of lidennina, GAEECE

Dr. T. Mud, Acaderny

Proprint Library. Hunganian Academy of Sa., HUNGARY

Dr. B. Dascupta, Sana inst of Nucteres Pryuices, INDIA

Dr. P. Kow, Inst. for Pinsma Research, INDIA

Dr. P. Rosenwe, leread inst of Technology, ISAAEL

Librarien, Intomational Conter for Theo Physica, ITALY

Wisc C. De Palo, Associazion EUPATOAHENEA, ITALY

Dr. G. Groses, Istiuts a Finica ded Plaema, ITALYY

Prof. G. Roctenoni, letitus Ger lonizus Dal Cnr, ITAL.Y

Dr. H. Yernes, Toeriba Pas Dovel Center, JAPAN
Prot. I. Kawakemi, Hiroshmna Univ., JAPAN

Prof. K. Nishikawa, Hiroshima Univ., JAPAN

Director, Jepan Atomic Enaroy Recoarch Inst, JAPAN

Prot. S. Inoh, Kyuthu Liniv., JAPAN

Pascarch Into. CU., Nationa Instit. for Fusion Science, JAPAN

Prot. S. Tanakil, Kyoto Uriv., JAPAN

Libremy, Kyoto Univ., JAPAN

Prof. N. Inowe, Univ. of Takyo, LAPAN

Sncrocery. Plema Section, Eloctrobctinical Lab., JAPAN

5. Mori. Tectrricad Actvicor, LAEF, JAPAN

Dr. O. Mhend, Kunances inst. of Technobog, JAPAN

J. Hyeon-Sook, Korea Alomic Energy Alesearch Inst. KOAEA

D.I. Chai, Tho Korea Adv. Inat of Sai. \& Tech., KOREA

Prot. B.S. Liby, Uriv. of Weikalo, NEW ZEALAND

Int of Phyrica, Chinese Acad Sci PEOPLE'S REP. OF CHINA Libryy, Inst of Plasma Physics, PECPLE'S REP. OF CHINA

Tainghue Univ. Librery, PEOPLE'S REPUBLIC OF CHINA

2. Li, S.W. Inat Phymics, PEOPLE'S REPUBLIC OF CHINA

Prot. J.A.C. Cobral, Imstuto SUperior Tecnico, PORTUGAL.

Dr. O. Porrue, N I CUza Univ., ROMaNIA

Dr. J. de Vuliars, fusion Studiues, AEC, S. AFRICA

Prof. M.A. Hotborg, Univ. of Natal, S. AFRICA

Prot. D.E Kom, Pohang inat. of Sa. Tech., SO. KOFEA

Prot. C.I.E.M.A.T. Fusion Division Library, SPAIN

Dr. L STomb, URIV. Of UMEA, SWEDEN

Library, Royed inst. of Tectnology, SWEDEN

Prot. H. Whatmeon, Chadress Univ, of Tech., SWEDEN

Camro Phys. Des Plasmas, Ecolo Polytech, SWITZERLAND

Dibliothedx, Inst. Voor Plazma-Fyeica, THE NETHERLANDS

Asat Prot. Dr. S. Cakir, Modo East Tach. Univ., TURKEY

Dr. V.A. Gwukhith, Sai. Rese. Insc. Electrophys.I Apperatus, USSA

Dr. D.D. Ayutov, Siburien Branch of Acaderny of Sa., USSA

Dr. G.A. Elinew. I.V. Kurchator Irmit. USSR

Librevien, The Ukr.SSA Acederny of Sciences, USSA

Or. LM. Kowizhnykh, Inet, of Generd Phycics, USSR

Komfonctungsantage GmbH, Zantrabibthoth, W. GERMANY

Bibliothek Inst. For Piasmatorothung. W. GERMANY

Prof. K. Schinder, Ruhr-Universt Bochum, W. GERMANY

Dr. F. Wagrer, (ASOEX), Max-Plunck-Institut, W. GERMANY

Loram, Max-Plencik-Institut, W. GERMANY

Prof. A.K. Janev, Inat of Phyeios, YUGOSLAVIA 

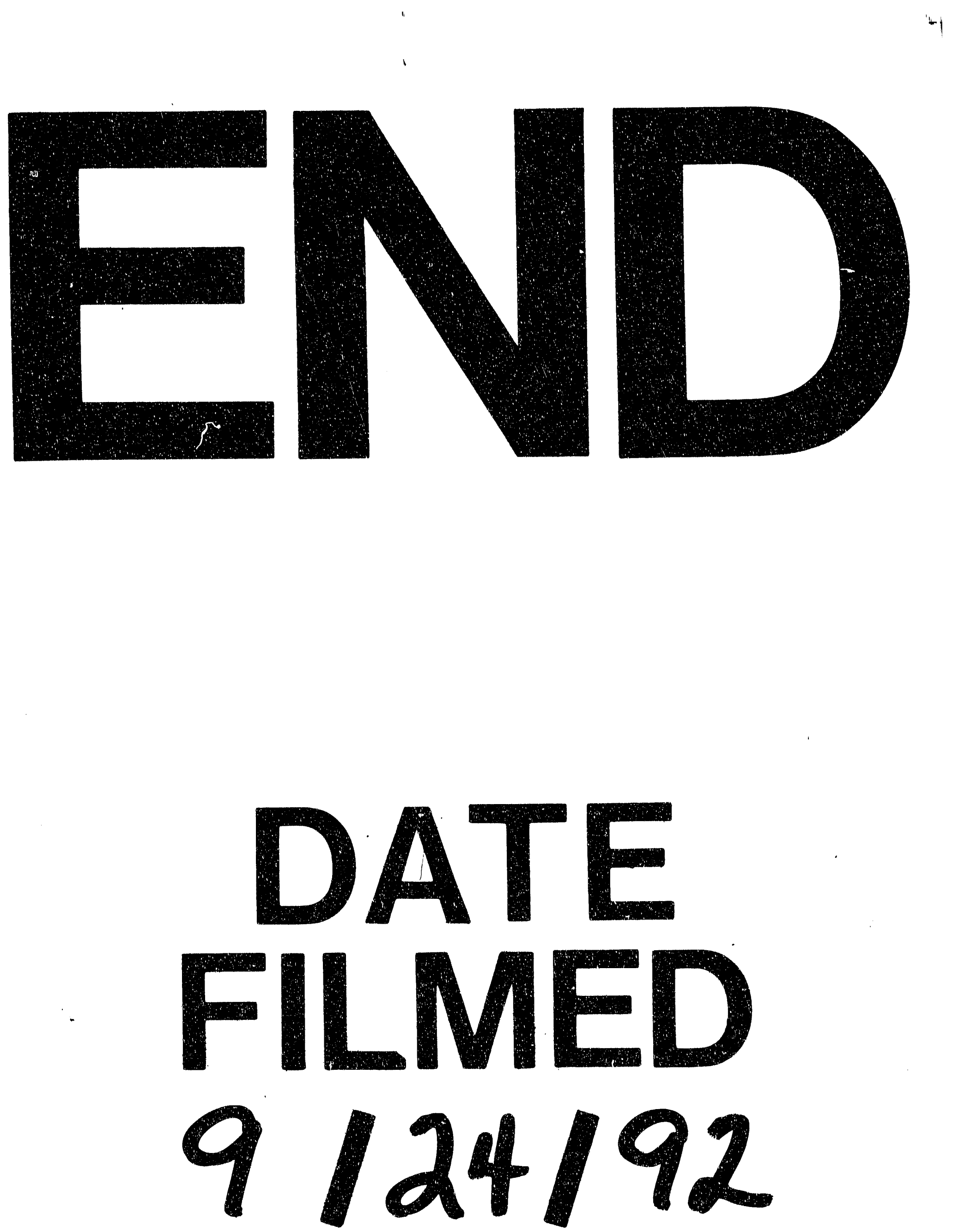
